\title{
La evaluacion docente en Anatomía Humana en enseñanzas remotas de emergencia
}

\section{Evaluation of human anatomy in emergency remote teaching}

\begin{abstract}
Alberto Garcia Barrios, Ana Isabel Cisneros Gimeno, Itziar Lamiquiz Moneo, Jaime Whyte Orozco.

Departamento de Anatomía e Histología Humanas. Facultad Medicina. Universidad de Zaragoza. España. Medical and Genetic Research Group (GIIS099) IIS Aragón. agarciab@unizar.es, 0000-0001-55603771; aicisner@unizar.es, 0000-0002-5494-343X; itziarlamiquiz@unizar.es, 0000-0002-9647-0108; jwhyte@unizar.es, 0000-0001-9372-4267.
\end{abstract}

* Correspondencia: aicisner@unizar.es

Recibido: 20/7/21; Aceptado: 29/10/21; Publicado: 2/11/21

\section{Resumen:}

El objetivo planteado en este estudio fue doble, evaluar la satisfacción del alumnado a realizar evaluación online, y las diferencias en esta según se realizase en casa o en el aula. El diseño y metodología se planteó mediante encuestas de satisfacción del tipo de evaluación y un análisis estadístico para valorar las diferencias en los resultados de esta según lugar de realización. La valoración del alumnado no muestra unanimidad (53\%) en considerar la evaluación online como un buen método de evaluación, aunque el 67\% y el 90\% (según curso y asignatura) usaría herramientas digitales para evaluar presencialmente. La reducción del tiempo y la conexión, son los principales inconvenientes valorados. Los resultados de la evaluación global no muestran cambios según sea presencial o a distancia. Por lo tanto, consideramos que la utilización de nuevas tecnologías para evaluar ha sido bien aceptada por los alumnos y por el profesorado, y no se observan resultados dispares realizándose presencial o a distancia.

Palabras clave: Anatomía, Evaluación, Online, Covid-19, Socrative ${ }^{\circledR}$

\begin{abstract}
:
The objective of the study was twofold: to evaluate student satisfaction with the online assessment, and the differences in this assessment according to whether it was carried out at home or in the classroom. The design and methodology were based on satisfaction surveys of the type of evaluation and a statistical analysis to assess the differences in the results according to the place where the evaluation was carried out. The students' evaluation does not show unanimity $(53 \%)$ in considering online evaluation as a good evaluation method, although $67 \%$ and $90 \%$ (depending on the course and subject) would use digital tools to evaluate face-to-face. The reduction of time and connection are the main disadvantages valued. The results of the global evaluation do not show changes depending on whether it is face-to-face or distance. Therefore, we consider that the use of new technologies for evaluation has been well accepted by students and teachers, and no different results are observed for face-to-face or distance learning.
\end{abstract}

Keywords: Anatomy, Evaluation, online, Covid-19, Socrative ${ }^{\circledR}$ 


\section{Introducción}

Durante el curso escolar 2019-2020, la comunidad educativa se vio bruscamente afectada por la pandemia asociada al SARS-COV2 (Covid-19), y obligada a modificar muchos aspectos relacionados con el proceso educativo a todos los niveles, desde educación infantil a educación superior. El cierre de las universidades de un día para otro, provocó la necesidad de utilizar nuevas tecnologías interactivas y virtuales para mantener los procesos de aprendizaje, y lo que es más importante, para realizar evaluaciones docentes no presenciales del alumnado.

La Evaluación educativa es un proceso que se inicia con el mismo aprendizaje y está presente a lo largo de todo el recorrido de un curso, una titulación, asignaturas o programas (1). En las últimas tres décadas la evaluación, como tema, como problema y como práctica, se ha convertido en un foco de atención para todos los involucrados (desde docentes al alumnado) en el campo de la Educación (2). Se podría definir como un método que se sustenta en el manejo de la información cualitativa y cuantitativa, para juzgar el logro y deficiencias del plan curricular, y tomar las decisiones relativas a ajustes, reformulación o cambios (3-7).

Para la realización del presente estudio se planteó el uso de la plataforma online Socrative ${ }^{\circledR}$, que además de poder ser utilizada como herramienta innovadora y que mejora el proceso de enseñanza aprendizaje en el transcurso de las clases a través de la interacción a tiempo real entre el docente y el alumnado, permite almacenar los resultados obtenidos por estos en los diferentes cuestionarios planteados (8-9). Este último aspecto, sumado a la posibilidad de su realización en versión online y poderse plantear como herramienta de evaluación a distancia, justificó el uso de esta herramienta para realizar el seguimiento del proceso de aprendizaje de los alumnos.

El objetivo que nos planteamos en este estudio fue doble: por un lado, analizar el efecto de la pandemia sobre la evaluación de contenidos y conocimientos, de la asignatura de Neuroanatomía y de otra de las asignaturas del área (Anatomía Humana I: Aparato Locomotor) para conocer el grado de satisfacción del alumnado y el profesorado, tras establecer una evaluación no presencial, con el mismo modelo de pruebas realizadas en el modo presencial; y por otro lado, según los resultados obtenidos en el cuestionario de satisfacción, plantear la evaluación docente del siguiente semestre, en la asignatura de Anatomía Humana II: Esplacnología, a través de plataformas online, observando las diferencias obtenidas cuando es planteada para su realización de manera presencial y/o a distancia.

\section{Métodos}

\section{OBJETIVO 1:}

La metodología planteada fue introducir la evaluación online, basada en preguntas tipo test, como se hubiera realizado en modo presencial, debido a la imposibilidad de realizarla en este formato. Este modelo de evaluación se planteó en el curso 2019-2020 para las asignaturas de Neuroanatomía Humana (impartida en el $2^{\circ}$ cuatrimestre de segundo curso), y Anatomía Humana I: Aparato Locomotor (impartida en el $2^{\circ}$ cuatrimestre de primer curso) ambas pertenecientes al Grado de Medicina de la Universidad de Zaragoza. Durante el desarrollo de este primer objetivo, la docencia se impartía única y exclusivamente vía online, debido al cierre de los centros educativos, consecuencia de la pandemia derivada de la Covid-19. 
En el caso de la asignatura de Aparato Locomotor, que cuenta con 168 alumnos, se planteó un cuestionario de 60 preguntas tipo test, que incluía 40 preguntas teóricas y 20 preguntas con imágenes ilustrativas a modo de "examen práctico", con las preguntas y respuestas de aparición aleatoria. El tiempo para resolver el cuestionario completo fue de 50 minutos.

En Neuroanatomía, que incluye a 114 alumnos, se evaluó exclusivamente la parte teórica de la asignatura, con un total de tres exámenes parciales, mediante un cuestionario de 20 preguntas tipo test, de aparición aleatoria, y un tiempo total de 15 minutos para su resolución.

Una vez llevadas a cabo las pruebas, se procedió a realizar una encuesta de valoración del método de evaluación por parte de los alumnos, de elaboración propia y diseñada específicamente y de propio para el desarrollo de este trabajo, valorando distintos aspectos relacionados con este método docente, y cuyos resultados se muestran en este manuscrito, mientras que la valoración por parte de los docentes se realizó mediante reunión de coordinación del profesorado involucrado.

La encuesta de valoración, respondida de manera voluntaria y anonimizada, estaba basada en 3 preguntas, siendo la primera de respuesta afirmativa/negativa, la segunda una cuestión con 5 opciones de respuesta del 1 al 5, indicando el grado de acuerdo o desacuerdo, siendo 1 "muy en desacuerdo" y 5 "muy de acuerdo", según la escala de Likert, y la última fue de respuesta múltiple, pudiendo seleccionar un numero variable de ítems según su valoración. Dicha encuesta de valoración se encuentra disponible como material suplementario (anexo 1), y no se planteó al comité de bioética de la Universidad de Zaragoza, al ser valoraciones personales y anónimas en relación al desarrollo de una experiencia docente.

\section{OBJETIVO 2:}

Se utilizó el mismo tipo de evaluación, mediante plataformas online que en el objetivo 1 , aunque en este caso la docencia se impartió presencial y en streaming al $50 \%$ consecuencia de las restricciones sanitarias. Se plantearon tres evaluaciones parciales durante el transcurso de la asignatura de Anatomía Humana II: Esplacnología, y se dividió a los 88 alumnos matriculados en esta asignatura en 2 subgrupos (subgrupo A y B) para realizar la evaluación a distancia o presencial de forma rotatoria en los parciales 1 y 2 respectivamente, y presencial para los dos subgrupos en el parcial 3. De esta manera, se pretende evaluar las diferencias en los resultados de evaluación, cuando esta se produce a distancia o presencialmente.

En el caso de la asignatura de Anatomía Humana II: Esplacnología, se planteó un cuestionario, tipo quizz, de 45 preguntas tipo test, que incluía 30 preguntas teóricas y 15 preguntas con imágenes ilustrativas a modo de "examen práctico", con las preguntas y respuestas ordenadas aleatoriamente. El tiempo para resolver el cuestionario completo fue de 40 minutos.

Una vez llevadas a cabo las pruebas relativas a cada parcial, se procedió a realizar una comparativa, mediante estudio estadístico con Rstudio (versión 3.5, Boston, Estados Unidos), de los resultados obtenidos por cada subgrupo y globalmente en cada una de las situaciones presentadas, y una encuesta al alumnado para valorar el grado de satisfacción con esta metodología a la hora de evaluar y la equidad en las tres pruebas realizadas. Los datos se expresan como la media \pm desviación estándar. El p valor fue calculado mediante el test T-Student. 
Al igual que en el objetivo 1, la encuesta al alumnado, de respuesta voluntaria y anonimizada, se basó en 3 preguntas con 5 opciones de respuesta del 1 al 5 , indicando el grado de acuerdo o desacuerdo, siendo 1 "muy en desacuerdo" y 5 "muy de acuerdo", según la escala de Likert, y una cuarta pregunta de respuesta afirmativa o negativa. Nuevamente, no se procedió a solicitar autorización al comité de bioética de la Universidad de Zaragoza, al tratarse de una valoración de una experiencia docente.

\section{Resultados}

\subsection{Objetivo 1.}

A nivel del alumnado, el cuestionario de evaluación planteado fue respondido por el $65 \%$ de estos. De sus respuestas se puede extraer que no hay unanimidad en considerar la evaluación online como un buen método de evaluar la asignatura, ya que el 53 \% están a favor de la evaluación online, mientras el 47\% restante están en contra.

Sin embargo, estos datos contrastan con la siguiente cuestión (Figura 1), sobre si realizar la evaluación docente mediante el uso de herramientas digitales, donde el $67 \%$ del alumnado estaría de acuerdo o totalmente de acuerdo en usar estos métodos digitales en modo presencial, el 11 \% estaría en contra y el 22\% restante no se decantaron por ninguna opción.

Por otro lado, cuando se consulta sobre los principales inconvenientes de la evaluación online (Figura 2), los resultados muestran que la reducción del tiempo de resolución (94\%) y los problemas de conexión (59\%) son las principales preocupaciones del alumnado, siendo la dificultad para el uso de estas plataformas (6\%) un factor poco preocupante para su resolución. Un $26 \%$ de los alumnos consideran que existen otros motivos como inconveniente.

\section{Inconvenientes realización evaluación online}

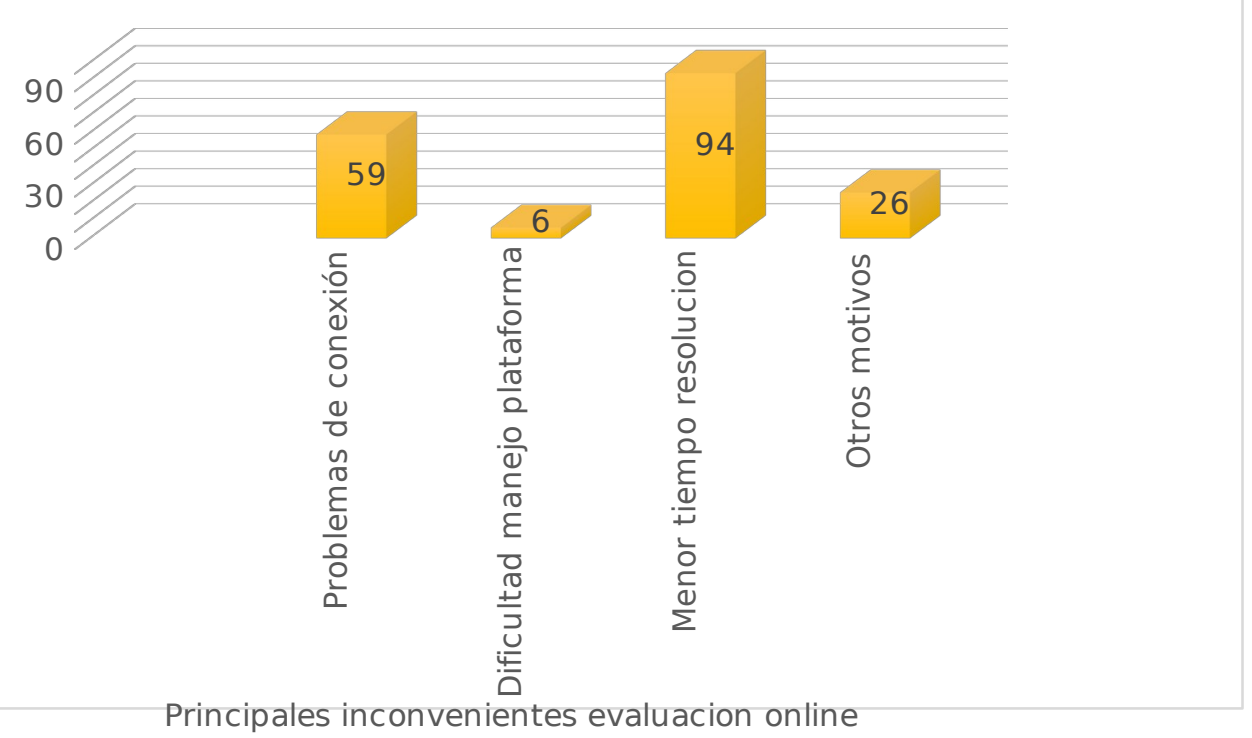

Figura 1. Valoración del alumnado para realización de la evaluación mediante plataformas digitales. 


\section{¿Consideras la herramienta Socrative ${ }^{\circledR}$ una herramienta de utilidad para la realización de la evaluación online de la asignatura?}

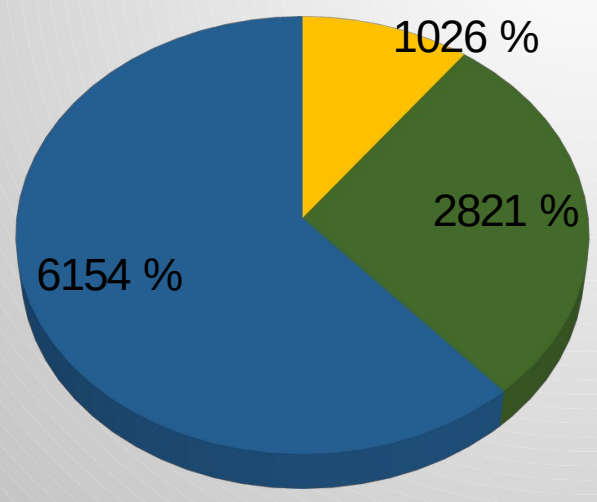

- Totalmente en desacuerdo

- En desacuerdo

Ni de acuerdo ni en desacuerdo

- De acuerdo

- Totalmente de acuerdo

Figura 2. Inconvenientes valorados por él alumnado en la evaluación online.

\subsection{Objetivo 2.}

Mediante la encuesta de evaluación al alumnado, respondida por el $78 \%$ de estos, se observó que el $90 \%$ de estos estaba de acuerdo en la utilidad de estas plataformas online para el desarrollo de la evaluación de la asignatura, independientemente de que esta se planteara de manera presencial o a distancia (Figura 3). Así mismo, el $100 \%$ del alumnado destacó la facilidad de uso de la plataforma en la que se realizó la evaluación, y la necesidad de extrapolarlo a otras asignaturas de la licenciatura (Figura 4).

Así mismo, se consultó por la dificultad a la hora de responder a los cuestionarios planteados en cada una de las pruebas, y el $67 \%$ de los alumnos estaban de acuerdo o completamente de acuerdo en que la dificultad de las pruebas había sido similar en los 3 ejercicios realizados, un $28 \%$ no estaba de acuerdo ni en desacuerdo en esta afirmación, y tan solo el 5\% del alumnado consideró estar de acuerdo en que había disparidad en los tipos de evaluación (Figura 5).

Basándonos en los resultados medios obtenidos en cada una de las pruebas, ya fuera de manera presencial o a distancia, se observa como uno de los subgrupos, en este caso el subgrupo B, obtiene siempre mejores resultados en las pruebas evaluativas, siendo estos significativos en los parciales 2 y 3 ( $p=0,001$ y $p=0,015$, respectivamente, Tabla 1 ).

Tabla 1. Resultados medios obtenidos por ambos subgrupos (1 y 2 ) en las diferentes pruebas de evaluación realizadas de manera presencial y/o a distancia.

\begin{tabular}{|l|c|c|c|}
\hline & Grupo A & Grupo B & $\boldsymbol{p}$ \\
\hline Primer parcial & $8,78 \pm 1,13 \#$ & $8,85 \pm 0,84^{\wedge}$ & 0,720 \\
\hline Segundo parcial & $8,75 \pm 0,73 \wedge$ & $9,21 \pm 0,65 \#$ & 0,001 \\
\hline Tercer parcial & $8,11 \pm 1,18 \#$ & $8,67 \pm 0,95 \#$ & 0,015 \\
\hline
\end{tabular}

Notas: ^ Evaluación a distancia / \# Evaluación presencial

Así mismo, se obtuvieron diferencias en los resultados de la evaluación según el lugar de realización de la prueba (en clase o en el aula), siendo mejores cuando estas se 
realizaban en el ámbito de clase, aunque solamente significativas para el subgrupo B $(p=0,016$, Tabla 2).

Tabla 2. Comparativa de los resultados de cada subgrupo en función del lugar de realización de la prueba evaluativa.

\begin{tabular}{|l|c|c|c|}
\hline & Clase & Casa & p \\
\hline Grupo A & $8,78 \pm 1,13$ & $8,75 \pm 0,73$ & 0,138 \\
\hline Grupo B & $9,21 \pm 0,65$ & $8,85 \pm 0,84$ & 0,016 \\
\hline
\end{tabular}

Por último, se evaluó independientemente del grupo, la media de notas de todos los parciales en función del lugar de realización, observándose que los resultados no son significativamente mejores cuando la evaluación se realiza presencial en el aula o vía telemática en casa ( $\mathrm{p}=0,369$, Tabla 3$)$.

Tabla 3. Comparativa global de los resultados obtenidos en las pruebas de evaluación presenciales o por vía telemática.

\begin{tabular}{|l|c|c|c|}
\hline & En casa & En clase & $\boldsymbol{p}$ \\
\hline Notas de ambos grupos & $8,79 \pm 0,77$ & $8,65 \pm 0,88$ & 0,369 \\
\hline
\end{tabular}

A nivel del profesorado, en reunión de coordinación de ambas asignaturas vía telemática, la valoración de los profesores fue unánime, considerando que la evaluación mediante el uso de dispositivos electrónicos en plataformas online es un método que puede implantarse en la docencia universitaria de aquellas asignaturas con evaluación mediante cuestionarios tipo test, en sustitución a la evaluación con papel, permitiendo obtener un feedback de los resultados prácticamente en el momento, disminuyendo así el tiempo de espera en la resolución para los alumnos, y reduciendo el gasto de papel, lo que supone un ahorro económico para los centros y un menor desgaste medioambiental. Por otro lado, pese a estar de acuerdo en el uso de este tipo de evaluación, consideramos que es necesario al menos igualar los tiempos de evaluación a las pruebas escritas, siempre y cuando estas se realicen de manera presencial, y dotar al alumnado de una buena conectividad.

\section{¿Consideras fácil el uso de la pla- taforma Socrative ${ }^{\circledR}$ y que debería extrapolarse a otras asignaturas?}

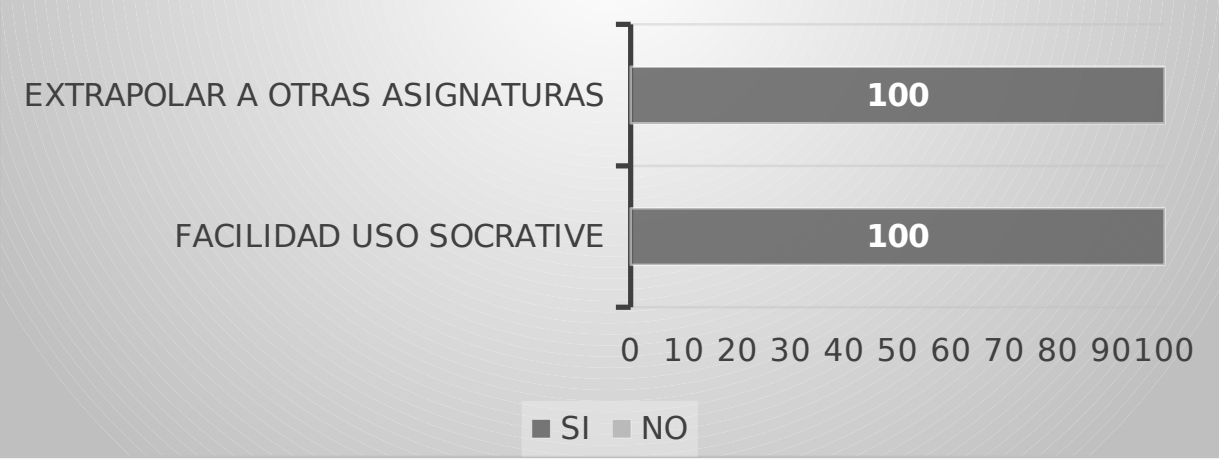

Figura 3. Evaluación de la herramienta Socrative para la realización de la evaluación online de la asignatura. 


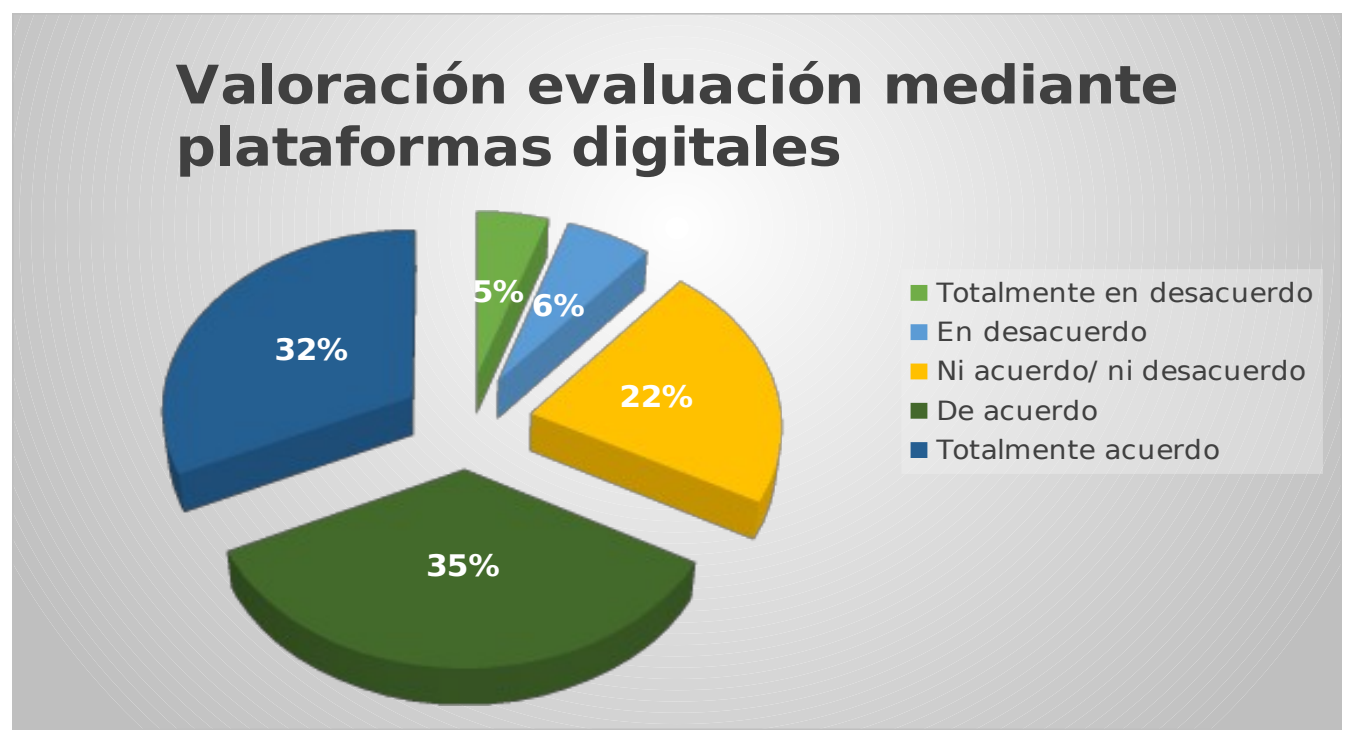

Figura 4. Valoración del alumnado en la facilidad de uso de Socrative y la posibilidad de extrapolarla a otras asignaturas.

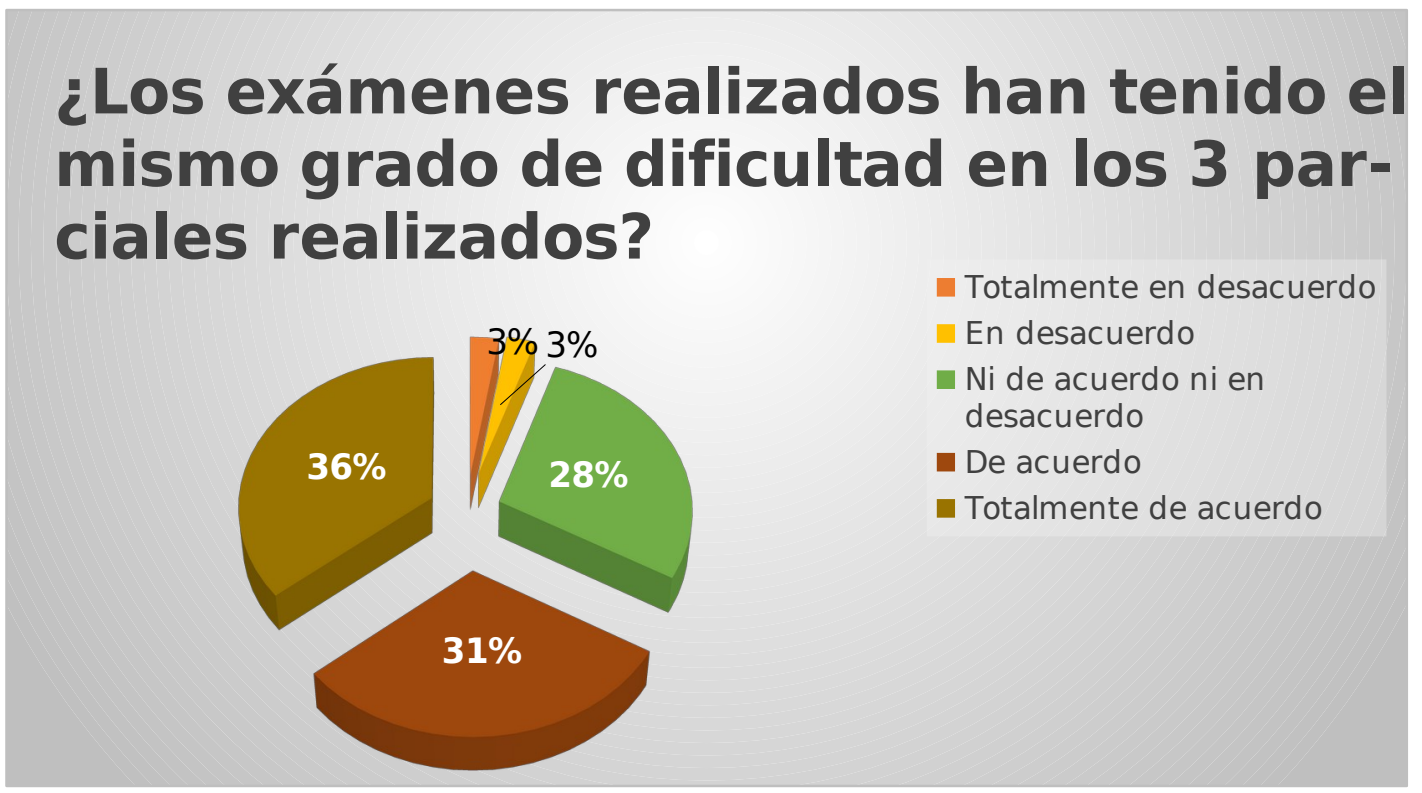

Figura 5. Respuesta de los alumnos al evaluar el grado de dificultad en las diferentes pruebas de evaluación.

\section{Discusión}

Durante la última década se ha llevado a cabo una transición gradual del aprendizaje tradicional presencial hacia el aprendizaje a distancia y este cambio ha sido especialmente acusado tras la pandemia de COVID-19. Son numerosos los artículos que han analizado la eficiencia del aprendizaje online en alumnos universitarios, incluyendo artículos que analizan el impacto del aprendizaje a distancia en el área de Anatomía (10-11). Sin embargo, son muy pocos los artículos que han realizado una comparativa entre la evaluación docente en el aula frente a la evaluación docente a distancia (12-13). Nuestros resultados han mostrado que no existen diferencias significativas en los resultados obtenidos de los exámenes realizados independientemente de que la evaluación se realice de manera presencial o a distancia. Estas mismas conclusiones indicaron Hakami y colaboradores (13), quienes mostraron que los conocimientos y destrezas obtenidos por 
parte de 92 alumnos que recibieron en un curso virtual de odontología en comparación con 106 alumnos que desarrollaron el mismo curso en la modalidad presencial eran los mismos. En la misma línea, Chang y colaboradores (12) también mostraron, a partir de los resultados obtenidos con 34 alumnos que recibían educación dental parte presencial y parte a distancia, que los resultados tras las evaluaciones correspondientes no diferían en función de donde se hubiera realizado el examen.

Las técnicas de evaluación van a estar condicionadas por el tipo de pruebas que se van a llevar a cabo, así como por los objetivos a alcanzar. En los modelos más tradicionales, la evaluación se asocia con pruebas escritas formales muy estructuradas en un tiempo determinado, ya sea en prueba oral o escrita (abierta o cerrada) (14), con las que no siempre se consigue apreciar totalmente el conocimiento del alumno. Por otro lado, el uso de las TIC, en materia educativa en general, y en la evaluación en particular, han abierto un nuevo abanico de posibilidades en la forma de evaluar, aunque a priori ello suponga un trabajo más laborioso para el docente (15), pero que facilita la administración de la información evaluativa, reduce el coste económico, el impacto medioambiental, el tiempo de corrección y permite hacer uso de los resultados obtenidos tantas veces como sea necesario. Además, la aceptación de estas plataformas como herramienta de evaluación es mejor valorada cuando han tenido contacto con ellas previamente.

Tal y como plantean también otros autores $(8,16)$, el uso de este tipo aplicaciones, es sencillo para el docente a la hora de realizar los cuestionarios online, y para el alumno, pues tan solo necesita introducir el código del "aula" donde se ha generado el cuestionario, administrado por el docente, y su nombre de usuario para poder ser identificado. Asimismo, estos cuestionarios online permiten evaluar diversos contenidos educativos, gestionar el flujo, el orden de las preguntas y respuestas, pudiendo generarlas de manera aleatoria y determinar la duración del cuestionario.

Además, la aplicación también permite el seguimiento del desarrollo de la prueba, ver las respuestas, marcando en distinto color si son correctas o no, e ir mostrando el porcentaje de la prueba ya realizado.

Al finalizar se pueden tener los resultados almacenados en el apartado Informes, en formato Excel ${ }^{\circledR}$, para poder visualizarlos posteriormente e incluso obtener un resumen de los mismos. En este resumen se recoge el cómputo de respuestas acertadas y fallidas, pudiendo ser individualizado para cada alumno o para cada una de las preguntas. También permite enviar los resultados a los alumnos por correo electrónico.

Sin embargo, esta evaluación únicamente online y no presencial, que a priori nos muestra tantas ventajas, no permite asegurar la identidad del alumno, ni estar seguros de que este está haciendo la prueba de forma individualizada, lo que podría conducir a la generación de situaciones poco responsables por parte del mismo, y al desarrollo de formas varias de picaresca. Esto lleva a que hoy en día diversas universidades a distancia, en las que la docencia se desarrolla en formato e-learning, opten por la evaluación presencial, como única forma posible de la misma. Sin embargo, esta situación podría reducirse realizando la evaluación de manera presencial a través de plataformas online, donde como hemos podido observar se obtienen resultados similares a los que se obtienen cuando la evaluación se realiza a distancia.

\section{Conclusiones}

- La utilización de nuevas tecnologías como método de evaluación ha sido en general bien aceptado por los alumnos y por el profesorado, haciendo uso de la aplica- 
ción Socrative ${ }^{\circledR}$.

- Estas aplicaciones permiten reducir el coste económico y el tiempo de corrección.

- Es necesario disponer de una buena conectividad y no reducir el tiempo de realización de las pruebas.

- La evaluación en dispositivo online de manera presencial, permite evitar el uso fraudulento de estas.

Material suplementario: anexo 1.

Contribuciones de los autores: Todos los autores han participado de forma activa en la elaboración de las encuestas de valoración y realización del análisis estadístico.

Financiación: Este trabajo no ha contado con ninguna financiación.

Declaración de conflicto de interés: Los autores declaran no tener ningún conflicto de intereses.

\section{Referencias}

1. Mora Vargas, A. I. La Evaluación Educativa: Concepto, Períodos y Modelos. Actual. Investig. En Educ. 2011, 4. https://doi.org/10.15517/aie.v4i2.9084.

2. Beltrán, M. R.; Schmelkes, S.; Díaz-Barriga, Á. La Evaluación Educativa1. Perfiles Educ. 2014. https://doi.org/10.1016/s0185-2698(14)70645-2.

3. Aparicio Gómez, W.; Aparicio Gómez, O. Y. Evaluación En Entornos Digitales; 2020. https://doi.org/10.13140/RG.2.2.26399.18080/1.

4. Dorrego, E. Educación a distancia y evaluación del aprendizaje. Rev. Educ. Distancia RED 2016, No. 50. https://doi.org/10.6018/red/50/12.

5. García-Peñalvo, F. J. Evaluación Del Aprendizaje En Entornos Virtuales y Remotos. Webinar emitido el 23 de junio de 2020. https://zenodo.org/record/3905200\#.YXVAZL9BxH4

6. Grande de Prado, M.; García Peñalvo, F. J.; Corell, A.; Abella García, V. La Virtualización Causada Por El Covid-19: Recomendaciones Para La Evaluación. Ensinar Avaliar E Aprender No Ensino Super. Perspetivas Int. 2020, 231-250.

7. Grande de Prado, M.; García Peñalvo, F. J.; Corell, A.; Abella-García, V. Evaluación En Educación Superior Durante La Pandemia de La COVID-19. Campus Virtuales 2021, 1 (10), 49-58.

8. Paz-Albo, J., \& Hervás, A. (2018). Socrative como herramienta de innovación en la educación superior. In I. Arco and P. Silva (Eds.), Tendencias nacionales e internacionales en organización educativa: entre la estabilidad y el cambio. Versión electrónica, Apartado I, cap. 27, pp. 18-22. Madrid: Wolters Kluwer. https://www.researchgate.net/publication/331477886_Socrative_como_herramienta_de_innovacion_en_1 a_educacion_superior

9. Frías, M.; Arce, C.; Flores-Morales, P. Uso de La Plataforma Socrative.Com Para Alumnos de Química General. Educ. Quim. 2015, 27. https://doi.org/10.1016/j.eq.2015.09.003.

10. Sadeesh, T.; Prabavathy, G.; Ganapathy, A. Evaluation of Undergraduate Medical Students' Preference to Human Anatomy Practical Assessment Methodology: A Comparison between Online and Traditional Methods. Surg. Radiol. Anat. SRA 2021, 43 (4), 531-535. https://doi.org/10.1007/s00276-020-02637-x.

11. Suárez-Escudero, J. C.; Muñoz, L. J. B.; Jurado, M. C. P.; Carmona, E. P. A.; Sánchez, A. J. U.; Burgos, S. R.; Gutiérrez, C. A. B.; Morales, J. L. F. Percepción de los estudiantes sobre adaptaciones virtuales en cursos de anatomía humana por la contingencia SARS-CoV-2. Acad. Virtualidad 2021, 14 (1), 151-168. https://doi.org/10.18359/ravi.5275.

12. Yu-Fong Chang, J.; Wang, L.-H.; Lin, T.-C.; Cheng, F.-C.; Chiang, C.-P. Comparison of Learning Effectiveness between Physical Classroom and Online Learning for Dental Education during the COVID19 Pandemic. J. Dent. Sci. 2021, 16 (4), 1281-1289. https://doi.org/10.1016/i.jds.2021.07.016.

13. Hakami, Z. Comparison between Virtual and Traditional Learning Methods for Orthodontic Knowledge and Skills in Dental Students: A Quasi-Experimental Study. Healthc. Basel Switz. 2021, 9 (9), 1092. https://doi.org/10.3390/healthcare9091092. 
14. Rodríguez Gómez, H. M.; Salinas Salazar, M. L. La Evaluación Para El Aprendizaje En La Educación Superior: Retos de La Alfabetización Del Profesorado. Rev. Iberoam. Eval. Educ. 2020, 13 (1), 111. https://doi.org/10.15366/riee2020.13.1.005.

15. Brown, S. A.; Glasner, A. Evaluar En La Universidad: Problemas y Nuevos Enfoques. Evaluar En Univ. Probl. Nuevos Enfoques 2003 ISBN 84-277-1422-X Págs 61-74 2003. https://dialnet.unirioja.es/servlet/libro? codigo $=2977$

16. Beltran, L. M. G.; Mamani, E. U. Socrative como una herramienta de evaluación permanente en el proceso de los aprendizajes. 37. Tesis de Investigación, 2020. http://repositorio.usil.edu.pe/handle/USIL/9838

(C) 2021 Universidad de Murcia. Enviado para su publicación en acceso abierto bajo los términos y condiciones de la licencia Creative Commons Reconocimiento-NoComercial-

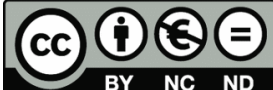

Sin Obra Derivada 4.0 España (CC BY-NC-ND)

(http:/ / creativecommons.org/licenses/by/4.0/).

Anexo 1. Cuestionario planteado a los alumnos.

\begin{tabular}{|c|c|c|}
\hline \multirow{3}{*}{ Objetivo 1} & Pregunta 1 & $\begin{array}{l}\text { ¿Estás de acuerdo en realizar la evaluación de la asignatura vía } \\
\text { online? }\end{array}$ \\
\hline & Pregunta 2 & $\begin{array}{l}\text { ¿Consideras factible realizar la evaluación, de al menos la parte } \\
\text { teórica de la asignatura, mediante el uso de dispositivos electrónicos } \\
\text { en plataformas online? }\end{array}$ \\
\hline & Pregunta 3 & $\begin{array}{l}\text { ¿Cuáles consideras que son los principales inconvenientes de realizar } \\
\text { una evaluación online? }\end{array}$ \\
\hline \multirow{3}{*}{ Objetivo 2} & Pregunta 1 & $\begin{array}{l}\text { ¿Consideras la herramienta socrative una herramienta de utilidad } \\
\text { para la realización de la evaluación online de la asignatura? }\end{array}$ \\
\hline & Pregunta 2 & $\begin{array}{l}\text { ¿Crees que el uso de la plataforma socrative para la evaluación } \\
\text { docente es de fácil uso para el alumnado? }\end{array}$ \\
\hline & Pregunta 3 & $\begin{array}{l}\text { ¿Los exámenes realizados han tenido el mismo grado de dificultad en } \\
\text { los } 3 \text { parciales realizados? }\end{array}$ \\
\hline
\end{tabular}

